\title{
Expression and distribution of HIF-1 $\alpha$, HIF-2 $\alpha$, VEGF, VEGFR-2 and HIMF in the kidneys of Tibetan sheep, plain sheep and goat
}

\author{
K. Yang ${ }^{1 *}$, Z. Zhang ${ }^{1 *}$, Y. Li ${ }^{1 *}$, S. Chen ${ }^{1}$, W. Chen ${ }^{1}$, H. Ding ${ }^{1}$, Z. Tan ${ }^{1}$, Z. Ma², Z. Qiao ${ }^{3}$ \\ ${ }^{1}$ College of Life Science and Engineering, Northwest Minzu University, Lanzhou, P.R. China \\ ${ }^{2}$ Key Laboratory of Biotechnology and Bioengineering of State Ethnic Affairs Commission, \\ Biomedical Research Centre, Northwest Minzu University, Lanzhou, P.R. China \\ ${ }^{3}$ Gansu Tech Innovation Centre of Animal Cell, Biomedical Research Centre, Northwest Minzu University, \\ Lanzhou, P.R. China
}

[Received: 19 December 2019; Accepted: 29 January 2020]

Background: The objective of this study was to detect the expression and distribution characteristics of five proteins (the hypoxia-inducible factor 1alpha [HIF-1 $\alpha$ ], HIF-2 $\alpha$, vascular endothelial growth factor [VEGF], VEGF-2 receptor [VEGFR-2] and hypoxia-induced mitogenic factor [HIMF]) in kidney of Tibetan sheep, plain sheep and goat. The results will provide the basic information for the comparative study of sheep breeds living at different altitudes.

Materials and methods: The kidney tissues were collected from healthy adult Tibetan sheep, plain sheep and goats and made into paraffin sections. Histological characteristics were assessed by haematoxylin and eosin staining. Expressions of HIF-1 $\alpha$, HIF-2 $\alpha$, VEGF, VEGFR-2 and HIMF proteins were measured by immunohistochemical staining.

Results: Immunohistochemistry results showed that the positive expression signals of HIF-1 $\alpha$, HIF-2 $\alpha$, VEGF and VEGFR-2 were detected in epithelial cells of renal tubules and collecting tubules, renal corpuscles in the kidneys of the three sheep breeds. Positive expression signals of HIMF were detected in epithelial cells of proximal tubules and distal tubules in Tibetan sheep and epithelial cells of distal tubules in goat. Immunostaining intensity of HIF-1 $\alpha, H I F-2 \alpha, V E G F$ and VEGFR-2 proteins in Tibetan sheep was significantly higher than that in both plain sheep and goat $(p<0.05)$. Immunostaining intensity of HIMF in Tibetan sheep was higher than goat $(p<0.05)$. Positive expression signals of HIMF were not detected in plain sheep.

Conclusions: The expression and distribution characteristics of HIF-1 $\alpha, H I F-2 \alpha$, VEGF, VEGFR-2 and HIMF in the studied kidney tissues suggested that these proteins may be related to the physiological regulation of Tibetan sheep kidney in hypoxia environment, and therefore might be important regulating proteins for Tibetan sheep to adapt to high altitude hypoxia environment. (Folia Morphol 2020; 79, 4: 748-755)

Key words: HIF-1 $\alpha$, HIF-2 $\alpha$, VEGF, VEGFR-2, HIMF, Tibetan sheep, kidney, immunohistochemistry

Address for correspondence: Dr. Z. Qiao, Gansu Tech Innovation Centre of Animal Cell, Biomedical Research Centre, Northwest Minzu University, Lanzhou, 730030, P.R. China, tel: +86 13639315431, e-mail: 670267497@qq.com

*These authors contributed equally to this article and share first authorship. 


\section{INTRODUCTION}

Hypoxia-inducible factor (HIF) pathway is a defence mechanism evolved to against hypoxia [5]. The HIFs are heterodimeric proteins that regulate the adaptive response to reduced oxygen availability at the cellular and organismal level [17]. HIFs consist of an $\mathrm{O}_{2}$-sensitive alpha subunit (HIF- $\alpha$ ) which is strongly induced following exposure to hypoxic conditions, and a constitutively expressed beta-subunit (HIF- $\beta$ ) which remains largely unaffected by changes of $\mathrm{O}_{2}$ level [11]. Among the three HIF- $\alpha$ isoforms (HIF-1 $\alpha$, HIF- $2 \alpha$ and HIF- $3 \alpha$ ), HIF- $1 \alpha$ and HIF- $2 \alpha$ were frequently studied [9]. Under normal oxygen conditions, HIF-1 $\alpha$ is highly unstable. Under hypoxia conditions, however, the half-life of HIF-1 $\alpha$ can be extended, leading to its accumulation and the formation of the HIF-1 complex. HIF- $2 \alpha$ is regulated in a similar way and shows high-level induction by hypoxia $[23,24]$. The two isoforms have similar functions in animals, such as both involving in metabolism and the process of angiogenesis and embryonic vascular development [11]. It is also showed that the reduction of their expression can decrease cell ability to survive [4]. For instance, the reduction of HIF- $1 \alpha$ can diminish cellular energy production, and the reduction of HIF- $2 \alpha$ can diminish hypoxia-induced pro-angiogenic factors and enhance anti-angiogenic factors [4]. Moreover Hahne et al. [4] indicated that HIF-1 $\alpha$ and HIF- $2 \alpha$ can coordinate with each other to adapt to hypoxia. In response to hypoxia, both HIF- $1 \alpha$ and HIF- $2 \alpha$ can regulate angiogenic genes such as vascular endothelial growth factor (VEGF) [11].

Vascular endothelial growth factor, a downstream target gene promoted by HIF activation, plays an essential role in the cellular adaption to hypoxia [11]. Hypoxia can also enhance VEGF expression, which has been found in many organs $[11,16]$. Kidney is susceptible to hypoxia, for its ability of angiogenesis can be affected under such condition [2]. VEGF enhances vascular permeability, stimulates angiogenesis, and promotes the delivery of blood to the hypoxic part, therefore can alleviate the tissue damage caused by hypoxia. VEGFR-2 is a receptor of VEGF and many of the biological activities of VEGF are mediated through VEGFR-2 in endothelial cells [14]. HIF- $1 \alpha$ and HIF-2 $\alpha$ have been demonstrated to increase target gene transcription (e.g. VEGF) in hypoxic cells. HIF-1 $\alpha$ expression regulates the production of VEGF, and HIF-2 $\alpha$ can activate VEGF preferentially [3]. Notably, renal tubular epithelial cells are also easy to be affected by hypoxia [26]. The hypoxia-induced mitogenic factor (HIMF) has been reported to enhance expression of VEGF in mouse lung epithelial cells [20].

Hypoxia is a key factor affecting organisms inhabiting such environment and plays an important role in organ diseases, such as renal injury and pulmonary hypertension [5, 7]. The ability of adaptation for mammalian cells to high-altitude and hypoxic conditions is an evolutionary modification and could result in considerable physiological changes related to animal viability, which have been demonstrated in some indigenous species living in this region, including Tibetan antelopes, gazelles and yaks $[15,18,30]$.

The Qinghai Tibet Plateau, the world's highest plateau, is famous for its hypoxia environment. Its atmosphere oxygen pressure is only $53-62 \%$ to sea level [10]. Tibetan sheep (Ovis aries) is a Chinese indigenous sheep breed and mainly distributed in the plateau and its adjacent areas. The sheep have been domesticated for more than $\mathbf{4 0 0 0}$ years and are well adapted to the hypoxic condition through natural selection [15], which makes it a perfect model to study the hypoxic-related mechanism. The current study used immunohistochemistry method to detect the expression and distribution characteristics of HIF- $1 \alpha$, HIF- $2 \alpha$, VEGF, VEGFR- 2 and HIMF in kidney from three sheep breeds dwelling in different altitudes (Tibetan sheep, plain sheep and goat). The results will also provide the baseline information to the understanding on mechanism of the stress response to high-altitude hypoxia environment.

\section{MATERIALS AND METHODS}

\section{Animal ethics}

The study was approved by the State Forestry Administration, and all procedures were performed in compliance with guidelines for the care and use of laboratory animals adopted by the Ministry of Science and Technology of the People's Republic of China.

\section{Materials}

Sheep individuals of 2-year-old male adults were collected in Hezuo City (with an altitude around 3,000 m, Gansu Province, China) for Tibetan sheep (Euler Tibetan sheep, $\mathrm{n}=5$ ) and Lanzhou City (with an altitude around 1,500 m, Gansu Province, China) for both plain sheep (small-tailed sheep, $n=5$ ) and goat (Boer goat, $n=5$ ) in September, 2019. Complied with local regulations, animals were euthanised with pentobar- 


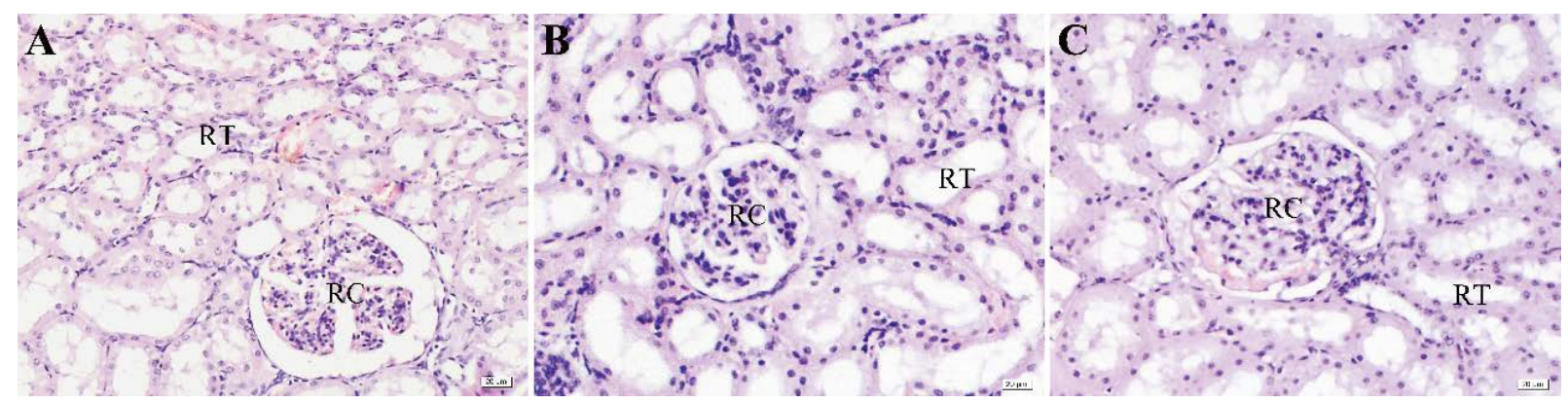

Figure 1. Histological observation of Tibetan sheep, plain sheep and goat kidneys; A. Tibetan sheep kidney; B. Plain sheep kidney; C. Goat kidney; RT — renal tubule; RC — renal corpuscle.

bital sodium (200 mg/kg, IV) at local slaughter houses. Kidney samples were collected and preserved in $4 \%$ paraformaldehyde for tissue fixation immediately after euthanasia.

\section{H\&E staining detection}

Fully fixed samples were embedded in paraffin and the tissue blocks then were sliced into $5 \mu \mathrm{m}$ sections for subsequent processes. Haematoxylin and eosin (H\&E) staining was used to observe the histological features of the samples.

\section{Immunohistochemistry detection}

Immunohistochemical staining was carried out based on Histostain ${ }^{\mathrm{TM}}$-Plus Kits (Bioss, China, SP-0023). Briefly, tissue sections were deparaffinised in xylene and rehydrated in different concentration gradient of alcohol. After being rinsed in phosphate buffered saline buffer (PBS), sections were autoclaved (15 min in a microwave oven) in $0.01 \mathrm{M}$ sodium citrate buffer (pH 6.0) for antigen retrieval. The endogenous peroxidase was inactivated using $3 \%$ hydrogen peroxide at $37^{\circ} \mathrm{C}$ for $10 \mathrm{~min}$. The sections were then incubated with anti-HIF- $1 \alpha$ polyclonal antibody, anti-HIF- $2 \alpha$ polyclonal antibody, anti-VEGF polyclonal antibody, anti-VEGFR-2 polyclonal antibody (Bioss, China, 1:200 dilution, bs-20398R, bs-1447R, bs-1665R and bs-0565R) and anti-HIMF polyclonal antibody (abcam, China, 1:200 dilution, ab39626) at $4^{\circ} \mathrm{C}$ overnight in a humid chamber. Antibody binding was coloured with DAB Substrate kit (Solarbio, China, DA1010) and tissue sections were counterstained with haematoxylin. All washing steps in-between were done in PBS. To assess the specificity of the immunolabelling, negative control slides were created using the bovine serum albumin as the primary antibody while all other steps and conditions remained the same.

\section{Statistical analysis}

Images of the stained tissue sections were observed and captured by a light microscope (Olympus CX31, Tokyo, Japan). Image-Pro Plus (Version 6.0, Media Cybernetics, Inc., Bethesda, MD, USA) was used to quantify the positive results of expression of HIF- $1 \alpha$, HIF- $2 \alpha$, VEGF, VEGFR-2 and HIMF. The measurement parameters included sum of the area and sum of the integrated optical density (IOD). SPSS software (Version 19.0, SPSS Inc., Chicago, USA) was used to analyse the statistical significance.

\section{RESULTS}

\section{Histological observation}

There was no significant difference observed in the renal structure among Tibetan sheep, plain sheep and goat (Fig. 1A-C). The uriniferous tubules of Tibetan sheep, plain sheep and goat consisted of renal corpuscles, renal tubules and collecting tubules.

In renal corpuscles, the glomeruli consisted of arterial capillaries which were coiled up into globular shape. Cup shape structured renal capsules were surrounded by central glomeruli; the outer epithelial cells of the renal capsules were surrounded by monolayer flat epithelial cells.

Renal tubules including proximal tubules, thin segments and distal tubules were surrounded by monolayer epithelial cells. The diameter of proximal tubules was relatively thick; its tube wall consisted of monolayer pyramidal cell with small and irregular diameter. The epithelial cells of distal tubules consisted of monolayer cubic cells with large and regular lumen. Thin segments consisted of monolayer flat cells with small diameter. The epithelial cells of collecting tubules were surrounded by monolayer cuboidal epithelium with clear cell boundary and large lumen. 

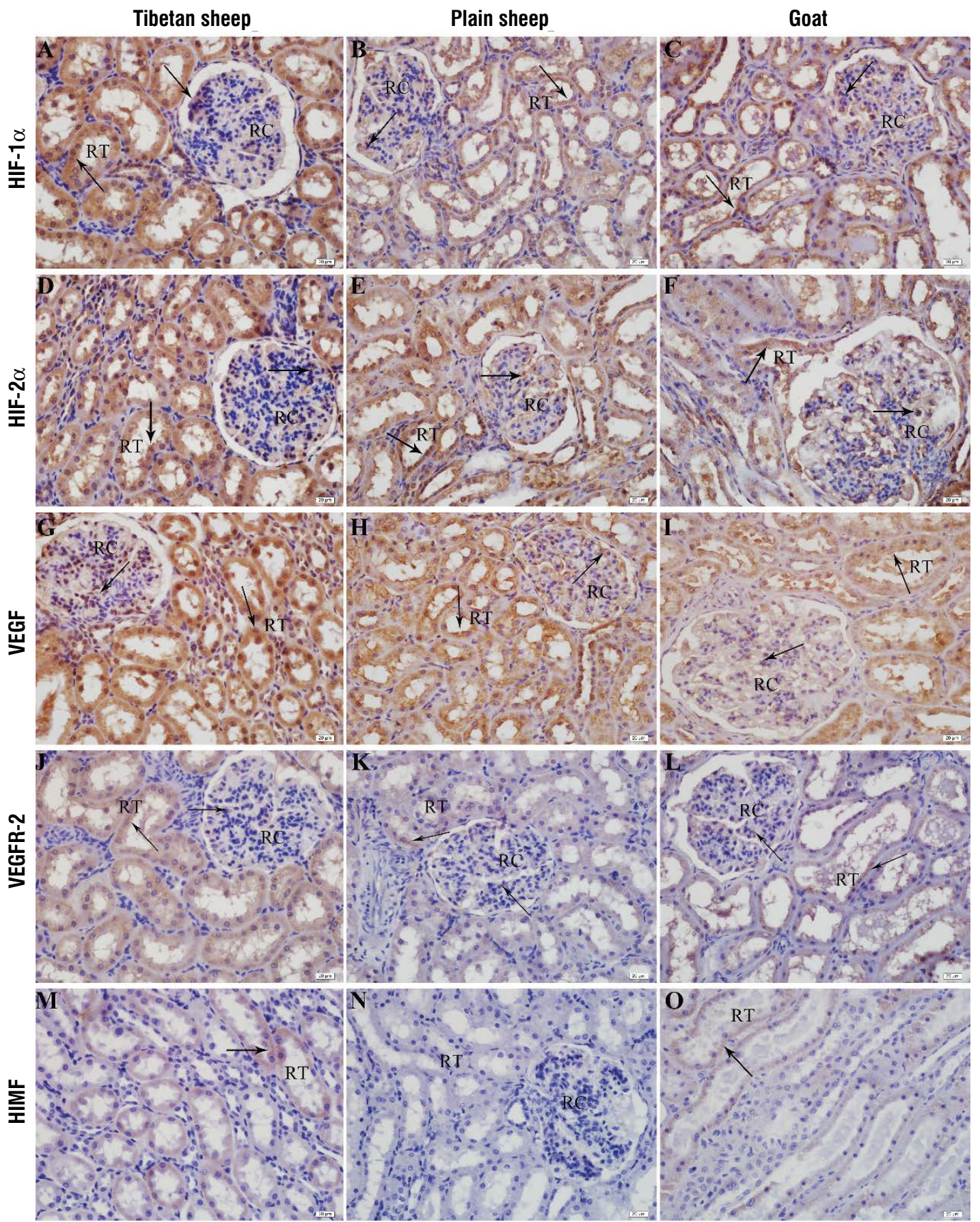

Figure 2. Representative images of immunostaining of the five proteins; A, B, C. HIF-1 $\alpha$ immunostaining for Tibetan sheep, plain sheep and goat, respectively; D, E, F. HIF-2 $\alpha$ immunostaining for Tibetan sheep, plain sheep and goat, respectively; G, H, I. VEGF immunostaining for Tibetan sheep, plain sheep and goat, respectively; J, K, L. VEGFR-2 immunostaining for Tibetan sheep, plain sheep and goat, respectively; M, N, 0. HIMF immunostaining for Tibetan sheep, plain sheep and goat, respectively. Arrows were denoted as the distribution of studied proteins in renal corpuscle (RC) and renal tubule (RT).

\section{Immunohistochemical localisations}

The positive expressions of HIF- $1 \alpha$, HIF- $2 \alpha$, VEGF, VEGFR-2 and HIMF were detected in Tibetan sheep in the positive control groups (Fig. 2A, D, G, J, M). Immunohistochemistry showed that HIF-1 $\alpha$, HIF-2 $\alpha$, VEGF and VEGFR-2 were mainly stained in the cytoplasm of renal tubule epithelial cells and collecting tubule epithelial cells, renal corpuscles in Tibetan sheep. The strong positive expression signals of HIF- $1 \alpha$, HIF- $2 \alpha$ and VEGF were found in the cytoplasm of distal tubule epithelial cells, proximal tubule epithelial cells and collecting tubule epithelial cells. The positive 
Table 1. Average integrated optical density values of HIF-1 $\alpha$, HIF-2 $\alpha$, VEGF, VEGFR-2 and HIMF in kidney of Tibetan sheep, plain sheep and goat (mean \pm standard deviation)

\begin{tabular}{lccccc}
\hline Species & HIF-1 $\alpha$ & HIF-2 $\alpha$ & VEGF & VEGFR-2 & HIMF \\
\hline Tibetan sheep & $0.0452 \pm 0.00526$ & $0.0393 \pm 0.00391$ & $0.0748 \pm 0.00939$ & $0.00882 \pm 0.00106$ & $0.000111 \pm 0.00000438$ \\
Plain sheep & $0.00934 \pm 0.00152$ & $0.0255 \pm 0.00775$ & $0.0313 \pm 0.00651$ & $0.000557 \pm 0.000274$ & - \\
Goat & $0.0160 \pm 0.00313$ & $0.0313 \pm 0.0107$ & $0.0356 \pm 0.00526$ & $0.000613 \pm 0.000289$ & $0.0000478 \pm 0.0000161$ \\
\hline
\end{tabular}

A

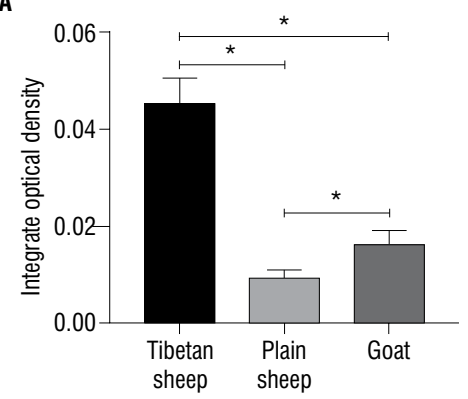

D

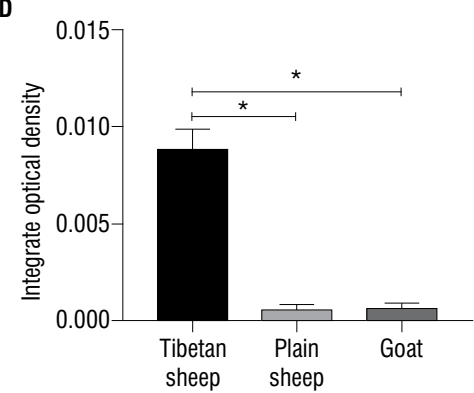

B

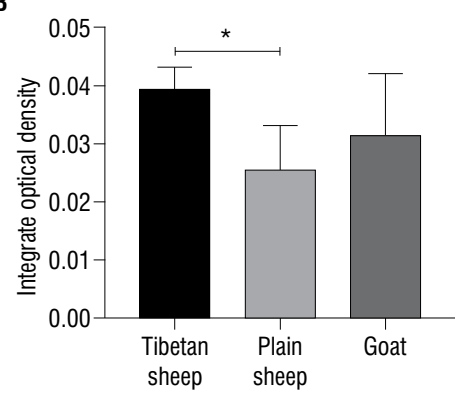

E

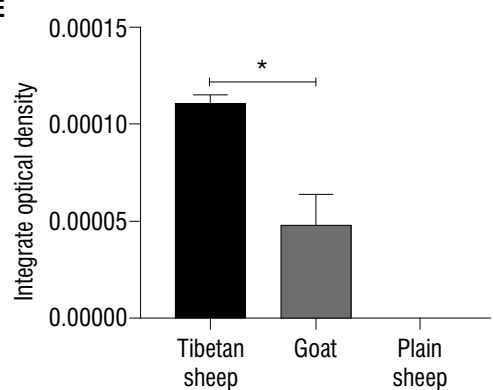

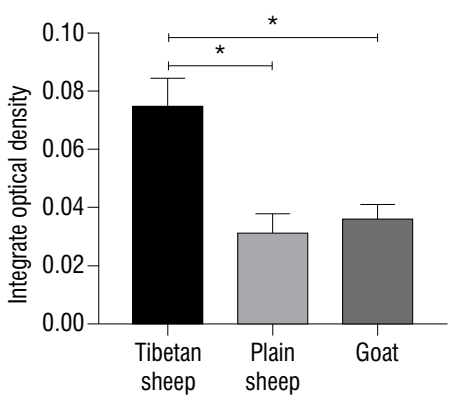

Figure 3. The comparison of integrated optical density (IOD) values of HIF-1 $\alpha$ (A), HIF-2 $\alpha$ (B), VEGF (C), VEGFR-2 (D), HIMF (E) among studied samples. Analysis of average IOD values of HIF-1 $\alpha$, HIF-2 $\alpha$, VEGF, VEGFR-2 was used one-way ANOVA, and the analysis of average IOD of HIMF was used independent sample t-test. The values were showed as mean \pm standard deviation; asterisk $(*)$ was indicated as significant difference $(p<0.05)$.

expression signals of HIMF were found in the cytoplasm of proximal and distal tubule epithelial cells.

For plain sheep, the positive expressions of HIF- $1 \alpha$, HIF-2 $\alpha$, VEGF, VEGFR-2 were all detected in the positive control groups (Fig. 2B, E, H, K), while HIMF showed no immunoactivity (Fig. $2 \mathrm{~N}$ ). Immunohistochemistry showed that HIF- $1 \alpha$, HIF- $2 \alpha$, VEGF and VEGFR-2 were clearly present in the cytoplasm of renal tubule epithelial cells, collecting tubule epithelial cells and renal corpuscles. The strong HIF- $1 \alpha$, HIF- $2 \alpha$, VEGF positive signals were found in the cytoplasm of distal tubule epithelial cells, proximal tubule epithelial cells and collecting tubule epithelial cells.

For samples of goat, the positive expressions of HIF-1 $\alpha$, HIF-2 $\alpha$, VEGF, VEGFR-2 and HIMF were detected in the positive control groups (Fig. 2C, F, I, L, O). Immunohistochemistry showed that HIF-1 $\alpha$, HIF- $2 \alpha$, VEGF and VEGFR-2 were expressed in the cytoplasm of renal tubule epithelial cells and collecting tubule epithelial cells, renal corpuscles. And strong HIF-1 $\alpha$, HIF- $2 \alpha$ and VEGF positive signals were found in the cytoplasm of distal tubule epithelial cells, proximal tubule epithelial cells and collecting tubule epithelial cells. HIMF immunoactivity showed in distal tubular epithelial cells.

\section{Optical density analysis}

Results from the averaged IOD (Table 1) showed that the immunostaining intensity of HIF- $1 \alpha$ was the highest in Tibetan sheep, followed by goat and plain sheep ( $p<0.05$, Fig. 3A). Tibetan sheep also had the highest immunostaining intensity of HIF-2 $\alpha$, VEGF, VEGFR-2 and HIMF (Fig. 3B-E). The results of the four proteins (HIF-1 $\alpha$, HIF-2 $\alpha$, VEGF, and VEGFR-2) for goat were generally higher than those in plain sheep with varied $p$ values (Table 1, Fig. 3). 


\section{DISCUSSION}

Oxygen is a vital substrate for aerobic organisms to maintain metabolism and physiological functions. Vertebrates have thus evolved complex respiratory and cardiovascular systems to ensure optimal $\mathrm{O}_{2}$ delivery to each cell $[11,28]$. Hypoxia, which is a biological restriction factor for most mammals, can cause various organ diseases such as renal injury and pulmonary hypertension $[5,25]$. However, organisms can be exposed to hypoxia for various reasons, such as hypoxic environment, hypoxaemia and ischaemia [5]. Therefore, adaptation to decreased oxygen tension is vital for organisms. The ability of mammals living in hypoxic environment such as the Qinghai-Tibetan Plateau to maintain homeostasis depends critically on appropriate respiratory regulation and blood pressure. In the meantime, low oxygen condition can activate cellular sensing mechanism with emphasis on restoring oxygen to hypoxia regions to maintain cell viability [9]. Accordingly, organisms have evolved a defence mechanism against hypoxia, the hypoxia-inducible factor pathway [5].

Hypoxia-inducible factor is a master gene switch for a variety of adaptive responses to hypoxia [11]. HIF- $1 \alpha$ and HIF- $2 \alpha$ are two heterodimeric hypoxia-inducible proteins regulated under hypoxic conditions [17]. They both can promote oxygen delivery and cellular adaptation to hypoxia via stimulating various cellular and tissue responses, including angiogenesis, erythropoiesis, anaerobic glucose metabolism and iron metabolism. Among these, angiogenesis, erythropoiesis, and anaerobic glucose metabolism are the most critical hypoxia responses in kidney injury and repair [19]. The three responses are regulated by VEGF, EPO and glycolytic genes, respectively. VEGF is a downstream target gene promoted by HIF; HIMF is also involved in the upregulation of VEGF expression for its angiogenic effect. In addition, VEGF and its receptor VEGFR-2 both play a key role in angiogenesis $[14,20]$.

Kidney, especially the renal medulla, is susceptible to hypoxia [26]. Notably, tubular domains in the renal outer medulla of kidney have the ability of glycolysis to generate ATP in the absence of oxygen [13]. HIF-1 $\alpha$ participates in the cellular process of transition from oxidative metabolism to glycolysis to induce the generation of ATP in an oxygen-independent manner [11]. Under normal conditions, HIF-1 is constantly expressed in the renal medulla but with a borderline hypoxic level for its stabilisation [13]. HIF-2 $\alpha$ is vital for renal cell development. Renal HIF-2 $\alpha$ is responsi- ble for the hypoxia-induced transcription of hypoxic response pathway, which is an important pathway for the mammals to response to hypoxia [8]. Liu et al. [10] reported that Tibetan antelope and Tibetan sheep both had higher expressions of HIF-1 $\alpha$ protein in the lung, cardiac muscle and skeletal muscle compared with plain sheep. In our study, HIF-1 $\alpha$ and HIF-2 $\alpha$ were highly expressed in Tibetan sheep kidney, comparing to that in goat and plain sheep. The results suggested that hypoxia environment may promote the expression of HIF-1 $\alpha$ and HIF-2 $\alpha$ in the kidney of Tibetan sheep. HIF- $1 \alpha$ and HIF- $2 \alpha$ can activate the expression of HIF-induced genes (e.g. EPO gene), further increasing erythrocytes, enhancing the capacity to delivery oxygen, promoting vascular regeneration and reconstruction. EPO thus plays an important role in adaption to hypoxia for plateau animals. Kidney is one of the main producers of EPO. EPO also functions on bone marrow stem cells whose increase promotes the regeneration of renal tubular epithelial cells and the kidney recovery from renal injury, alleviating renal damage caused by hypoxia $[11,12]$. Our kidney tissue sections demonstrated that both HIF- $1 \alpha$ and HIF- $2 \alpha$ were stained intensively in renal tubule epithelial cells and collecting tubule epithelial cells. Expression of HIF- $1 \alpha$ and HIF- $2 \alpha$ in Tibetan sheep was higher than goat and plain sheep, indicating that HIF- $1 \alpha$ and HIF- $2 \alpha$ were important proteins for its adaption to the high-altitude hypoxia environment.

Vascular endothelial growth factor and its receptor VEGFR-2 play a pivotal role in angiogenesis and cell proliferation. VEGF can promote blood flow by promoting the formation of blood vessels in the hypoxic area, thus reducing the tissue damage caused by hypoxia [5]. In endothelial cells, many of the biological activities of VEGF are mediated through VEGFR-2 [14]. Endothelial cells can obtain oxygen directly from red blood cells, but can also be affected by hypoxia [13]. Epithelial tubular cell is another type of cells that is affected by hypoxia besides endothelial and vascular cells [26]. In kidney tissue sections, we also observed that VEGF and VEGFR-2 were mainly stained in the renal corpuscles and epithelial cells in renal tubules and collecting tubules. Positive signals of VEGF and VEGFR-2 in Tibetan sheep kidney were stronger than plain sheep and goat, suggesting that VEGF and VEGFR-2 play an important role in reducing the effects of high-altitude hypoxia environment on the kidney of Tibetan sheep. It has been reported that VEGF is mainly produced by podocytes and renal tubular epi- 
thelial cells in the kidney, which is consistent with our results. Podocytes are indispensable components to ensure the integrity of glomerular filtration membrane structure, while epithelial cells in renal tubules maintain the structure and function of capillary network around renal tubules, resist interstitial fibrosis, and participate in the formation of endothelial cells [1]. Villegas et al. [21] found that VEGF and VEGFR-2 expressed in proximal tubular epithelial cell line in vitro, and confirmed that VEGF mainly functions by binding with VEGFR-2. In addition, VEGF produced by renal tubular epithelial cells can promote the proliferation of renal tubular cells by autocrine. These studies indicate that VEGF and VEGFR-2 are involved in the maintenance of renal structure and regulation of renal function. The high expression of VEGF and VEGFR-2 in Tibetan sheep kidney discovered in our study may be thus related to the adaptation of Tibetan sheep to high altitude hypoxia environment.

Study showed that mutant endothelial cells isolated from mutant mice exhibited defective hypoxic activation of VEGF and VEGFR-2 as well as impaired cell proliferation and migration [11]. The authors therefore suggested that HIF-1 $\alpha$ promoted an autocrine VEGF/ NEGFR-2 loop in endothelial cells, facilitating their functions in tissue angiogenesis [11]. HIMF can also directly stimulate the production of VEGF and promote angiogenesis through VEGF/VEGFR2 pathway. HIMF is a protein first found in pulmonary hypertension induced by hypoxia mouse model. It was reported as a lung-specific growth factor, involving in lung cell proliferation and regulation of compensatory lung growth [27]. HIMF has functions of angiogenic and vasoconstrictive [29]. In lung epithelial cells of mouse, HIMF enhances VEGF production in a PI-3K/Akt-NF-KB signalling pathway-dependent manner [20]. In addition, VEGF mRNA synchronously changes with HIF-1 $\alpha$ mRNA during hypoxia, which shows that enhanced expression of HIF-1 can activate VEGF gene transcription, increase VEGF production, promote vascular construction, drive blood to the hypoxic part of renal tubules, thus reduce renal injury $[6,12]$. Hoppeler and Vogt [6] reported that HIF-1 $\alpha$ mRNA was upregulated after 6 weeks of endurance training in hypoxia (equivalent to an altitude of $3,850 \mathrm{~m}$ ) in previously untrained subjects, and high intensity training in hypoxia further resulted in an increased expressions of VEGF mRNA, capillarity and myoglobin mRNA. This upregulation was showed to be independent of training intensity and not observed in subjects under similar conditions in normoxia. These findings have been further confirmed by later researcher [22]. The protective effects (e.g. alleviating renal injury) of HIF-1 $\alpha$, HIF-2 $\alpha$ and hypoxia-induced VEGF have been shown in chronic kidney disease [5].

In our study, HIMF was mainly expressed in distal tubule epithelial cells and proximal tubule epithelial cells for Tibetan sheep. HIMF was also expressed in distal tubule epithelial cells in goat, but no HIMF immunoactivity showed in plain sheep. Taking account of the findings from previous studies, along with the results of positive signals of HIF- $1 \alpha$, HIF- $2 \alpha$, HIMF, VEGF and VEGFR-2 showed in the kidney of Tibetan sheep, our study suggested that HIF- $1 \alpha$, HIF- $2 \alpha$, HIMF, VEGF and its receptor VEGFR-2 may be involved in the protection, structure maintenance and function regulation of Tibetan sheep kidney under hypoxia environment.

\section{CONCLUSIONS}

Our results indicated that expression and distribution characteristics of HIF- $1 \alpha$, HIF-2 $\alpha$, VEGF, VEGFR-2 and HIMF in the kidney of Tibetan sheep may be related to the physiological regulation of Tibetan sheep inhabiting hypoxic environment. They are thus considered as important potential regulating proteins in Tibetan sheep's adaptation to hypoxia environment. However, the underlying molecular mechanisms are not clear. This study is a preliminary experiment and the mechanism of such regulation in Tibetan sheep needs to be further explored. This study will also provide baseline information for comparison studies between sheep breed inhabiting arears with different altitude.

\section{Acknowledgements}

This study was supported by The National Natural Science Foundation of China (grant no. 31860687), The Fundamental Research Funds for the Central Universities for Northwest Minzu University (3192020004, 31920190020, 31920170177, 31920190004), Changjiang Scholars and Innovative Research Team in University of Ministry of Education, China (IRT_17R88).

\section{REFERENCES}

1. Brosius FC, Coward RJ. Podocytes, signaling pathways, and vascular factors in diabetic kidney disease. Adv Chronic Kidney Dis. 2014; 21(3): 304-310, doi: 10.1053/j. ackd.2014.03.011, indexed in Pubmed: 24780459.

2. Evans RG, Smith DW, Lee CJ, et al. What Makes the Kidney Susceptible to Hypoxia? Anat Rec (Hoboken). 2019 [Epub ahead of print], doi: 10.1002/ar.24260, indexed in Pubmed: 31566903.

3. Gordan JD, Bertout JA, Hu CJ, et al. HIF-2alpha promotes hypoxic cell proliferation by enhancing c-myc transcrip- 
tional activity. Cancer Cell. 2007; 11(4): 335-347, doi: 10.1016/j.ccr.2007.02.006, indexed in Pubmed: 17418410.

4. Hahne $M$, Schumann $P$, Mursell $M$, et al. Unraveling the role of hypoxia-inducible factor (HIF)- $1 \alpha$ and HIF- $2 \alpha$ in the adaption process of human microvascular endothelial cells (HMEC-1) to hypoxia: Redundant HIF-dependent regulation of macrophage migration inhibitory factor. Microvasc Res. 2018; 116: 34-44, doi: 10.1016/j.mvr.2017.09.004, indexed in Pubmed: 28993199.

5. Honda T, Hirakawa $Y$, Nangaku M. The role of oxidative stress and hypoxia in renal disease. Kidney Res Clin Pract. 2019; 38(4): 414-426, doi: 10.23876/j.krcp.19.063, indexed in Pubmed: 31558011.

6. Hoppeler $\mathrm{H}$, Vogt M. Hypoxia training for sea-level performance. Training high-living low. Adv Exp Med Biol. 2001; 502: 61-73, doi: 10.1007/978-1-4757-3401-0_6, indexed in Pubmed: 11950155.

7. Huang J, Frid M, Gewitz MH, et al. Hypoxia-induced pulmonary hypertension and chronic lung disease: caveolin-1 dysfunction an important underlying feature. Pulm Circ. 2019; 9(1): 2045894019837876, doi: 10.1177/2045894019837876, indexed in Pubmed: 30806156.

8. Kapitsinou PP, Liu Q, Unger TL, et al. Hepatic HIF-2 regulates erythropoietic responses to hypoxia in renal anemia. Blood. 2010; 116(16): 3039-3048, doi: 10.1182/ blood-2010-02-270322, indexed in Pubmed: 20628150.

9. Labrousse-Arias D, Castillo-González R, Rogers NM, et al. HIF-2 $\alpha$-mediated induction of pulmonary thrombospondin-1 contributes to hypoxia-driven vascular remodelling and vasoconstriction. Cardiovasc Res. 2016; 109(1): 115-130, doi: 10.1093/cvr/cvv243, indexed in Pubmed: 26503986.

10. Liu F, Wuren $T, M a L$, et al. [Genetic cloning and expression of hypoxia inducible factor 1 alpha in high altitude hypoxic adaptation species Tibetan antelope (Pantholops hodgsonii)]. Sheng Li Xue Bao. 2011; 63(6): 565-573, indexed in Pubmed: 22193452.

11. Majmundar AJ, Wong WJ, Simon MC. Hypoxia-inducible factors and the response to hypoxic stress. Mol Cell. 2010; 40(2): 294-309, doi: 10.1016/j.molcel.2010.09.022, indexed in Pubmed: 20965423.

12. Maxwell P. HIF-1: an oxygen response system with special relevance to the kidney. J Am Soc Nephrol. 2003; 14(11): 2712-2722, doi: 10.1097/01.asn.0000092792.97122.e0, indexed in Pubmed: 14569080.

13. Mimura I, Nangaku M. The suffocating kidney: tubulointerstitial hypoxia in end-stage renal disease. Nat Rev Nephrol. 2010; 6(11): 667-678, doi: 10.1038/nrneph.2010.124, indexed in Pubmed: 20877304.

14. Murphy JF, Fitzgerald DJ. Vascular endothelial growth factor induces cyclooxygenase-dependent proliferation of endothelial cells via the VEGF-2 receptor. FASEB J. 2001; 15(9): 1667-1669, doi: 10.1096/fj.00-0757fje, indexed in Pubmed: 11427521.

15. Niu L, Chen X, Xiao P, et al. Detecting signatures of selection within the Tibetan sheep mitochondrial genome. Mitochondrial DNA A DNA Mapp Seq Anal. 2017; 28(6): 801-809, doi: 10.1080/24701394.2016.1192614, indexed in Pubmed: 27937004.

16. Park SY, Jeong KJ, Lee J, et al. Hypoxia enhances LPA-induced HIF-1 alpha and VEGF expression: their inhibition by resveratrol. Cancer Lett. 2007; 258(1): 63-69, doi: 10.1016/j. canlet.2007.08.011, indexed in Pubmed: 17919812.
17. Patel SA, Simon MC. Biology of hypoxia-inducible factor-2alpha in development and disease. Cell Death Differ. 2008; 15(4): 628-634, doi: 10.1038/cdd.2008.17, indexed in Pubmed: 18259197.

18. Qiu Q, Zhang G, Ma T, et al. The yak genome and adaptation to life at high altitude. Nat Genet. 2012; 44(8): 946-949, doi: 10.1038/ng.2343, indexed in Pubmed: 22751099.

19. Shu S, Wang $Y$, Zheng M, et al. Hypoxia and hypoxia-inducible factors in kidney injury and repair. Cells. 2019; 8(3), doi: 10.3390/cells8030207, indexed in Pubmed: 30823476.

20. Tong $Q$, Zheng $L$, Lin Li, et al. VEGF is upregulated by hypoxia-induced mitogenic factor via the PI-3K/Akt-NF-kappaB signaling pathway. Respir Res. 2006; 7: 37, doi: 10.1186/1465-9921-7-37, indexed in Pubmed: 16512910.

21. Villegas G, Lange-Sperandio B, Tufro A. Autocrine and paracrine functions of vascular endothelial growth factor (VEGF) in renal tubular epithelial cells. Kidney Int. 2005; 67(2): 449-457, doi: 10.1111/j.1523-1755.2005.67101.x, indexed in Pubmed: 15673292.

22. Vogt M, Puntschart A, Geiser J, et al. Molecular adaptations in human skeletal muscle to endurance training under simulated hypoxic conditions. J Appl Physiol (1985). 2001; 91(1): 173-182, doi: 10.1152/jappl.2001.91.1.173, indexed in Pubmed: 11408428.

23. Wang GL, Jiang BH, Rue EA, et al. Hypoxia-inducible factor 1 is a basic-helix-loop-helix-PAS heterodimer regulated by cellular O2 tension. Proc Natl Acad Sci U S A. 1995; 92(12): 5510-5514, doi: 10.1073/pnas.92.12.5510, indexed in Pubmed: 7539918.

24. Wenger RH. Mammalian oxygen sensing, signalling and gene regulation. J Exp Biol. 2000; 203(Pt 8): 1253-1263, indexed in Pubmed: 10729275.

25. Will DH, Hicks JL, Card CS, et al. Inherited susceptibility of cattle to high-altitude pulmonary hypertension. J Appl Physiol. 1975; 38(3): 491-494, doi: 10.1152/jappl.1975.38.3.491, indexed in Pubmed: 238929.

26. Willam C, Ditting T, Veelken R, et al. Cardio-Renal Connection: The Role of Hypoxia and Oxidative Stress. Studies Renal Dis. 2010: 499-533, doi: 10.1007/978-1-60761-857-7_26.

27. Yamaji-Kegan K, Su Q, Angelini DJ, et al. Hypoxia-induced mitogenic factor has proangiogenic and proinflammatory effects in the lung via VEGF and VEGF receptor-2. Am J Physiol Lung Cell Mol Physiol. 2006; 291(6): L1159-L1168, doi: 10.1152/ajplung.00168.2006, indexed in Pubmed: 16891392.

28. Yuan G, Peng YJ, Reddy VD, et al. Mutual antagonism between hypoxia-inducible factors $1 \alpha$ and $2 \alpha$ regulates oxygen sensing and cardio-respiratory homeostasis. Proc Natl Acad Sci U S A. 2013; 110(19): E1788-E1796, doi: 10.1073/ pnas.1305961110, indexed in Pubmed: 23610397.

29. Zeng X, Zhu L, Xiao R, et al. Hypoxia-Induced mitogenic factor acts as a nonclassical ligand of calcium-sensing receptor, therapeutically exploitable for intermittent hypoxia-induced pulmonary hypertension. Hypertension. 2017; 69(5): 844-854, doi: 10.1161/HYPERTENSIONAHA.116.08743, indexed in Pubmed: 28348014.

30. Zhang $F$, Jiang Z. Mitochondrial phylogeography and genetic diversity of Tibetan gazelle (Procapra picticaudata): implications for conservation. Mol Phylogenet Evol. 2006; 41 (2): 313-321, doi: 10.1016/j.ympev.2006.05.024, indexed in Pubmed: 16837214. 